\title{
Endovascular management of cerebral vasospasm
}

\author{
Craig Kelman, M.D. and John Reavey-Cantwell, M.D., M.S. \\ VCU Medical Center - Department of Neurosurgery, Virginia Commonwealth University, Richmond, Virginia
}

\begin{abstract}
Cerebral vasospasm remains a feared complication from subarachnoid hemorrhage. Vasospasm typically occurs from three to fourteen days post-aneurysm rupture, with peak risk on Day 7. Up to $50 \%$ of patients with angiographic vasospasm will subsequently develop delayed cerebral ischemia; $15-20 \%$ of this subset will develop stroke or death despite maximal medical therapy. We define symptomatic vasospasm as the presence of neurological worsening after exclusion of other identifiable causes, such as seizure, hydrocephalus, intracerebral hemorrhage, or metabolic dysfunction. This video demonstrates the set-up and treatment protocol for endovascular intervention of symptomatic vasospasm not responsive to maximal medical management.
\end{abstract}

The video can be found here: http://youtu.be/q7YvxLzIDnU.

(http://thejns.org/doi/abs/10.3171/2014.V2.FOCUS14172)

\begin{tabular}{|c|c|c|}
\hline $\begin{array}{l}\text { KEY WORDS } \\
\text { endovascular }\end{array}$ & $\begin{array}{ll}\text { - } & \text { vasospasm } \\
\text { - } & \text { verapamil }\end{array}$ & $\begin{array}{c}\text { - } \\
\text { - } \\
\text { aneurysm }\end{array}$ \\
\hline
\end{tabular}

Manuscript submitted April 30, 2014.

Accepted May 29, 2014.

Please include this information when citing this paper: DOI: 10.3171/2014.V2.FOCUS14172.

Address correspondence to: Dr. John Reavey-Cantwell, VCU Medical Center, Virginia Commonwealth University, Ambulatory Care Center 6th Floor, Department of Neurosurgery, 417 North 11th Street, PO Box 980631, Richmond, Virginia 23298. email: jreaveycantwell@mcvh-vcu.edu. 